02

\title{
Исследование диэлектрических свойств полости кукурбитурила на основе эффекта сольватохромизма стирилового красителя при образовании комплекса включения
}

\author{
(C) А.С. Степко ${ }^{1}$, Н.А. Лобова ${ }^{1,2}$, П.В. Лебедев-Степанов ${ }^{1,3}$ \\ ${ }^{1}$ Центр фоотохимии РАН, \\ „Федеральный научно-исследовательский центр „Кристаллография и фотоника“ РАН“, \\ 119421 Москва, Россия \\ ${ }^{2}$ Московский фризико-технический институт (государственный университет), \\ 117303 Долгопрудный, Московской обл., Россия \\ ${ }^{3}$ Национальный исследовательский ядерный университет „МИФИ“, \\ 115409 Москва, Россия \\ e-mail: tousen@bk.ru
}

Поступила в редакцию 11.05.2018 г.

В окончательной редакции 17.09.2018 г.

Принята к публикации 04.12.2018 г.

\begin{abstract}
Предложен новый подход для теоретической оценки локальных диэлектрических свойств среды, в которую помещен органический краситель, работающий в качестве нанозонда. Метод апробирован для оценки диэлектрических свойств молекулы-,хозяина“ в супрамолекулярном комплексе включения по измеренному в эксперименте сдвигу оптического спектра поглощения молекулы-,гостя“ (красителя) при формировании комплекса. На основе модели Онзагера-Липтэя проведена теоретическая оценка диэлектрических свойств полости кукурбит[7]урила путем анализа экспериментально измеренного сдвига спектра поглощения дикатиона красителя 1-(3-аммонийпропил)-4-[(Е)-2-(3,4-диметоксифинил)-этенил-]-пиридиния при образовании комплекса включения с кукурбит[7]урилом в водном растворе. Для параметризации уравнения ОнзагераЛиптэя были экспериментально измерены положения максимумов спектров поглощения рассматриваемого красителя в следующих растворителях: метанол, этанол, 1-пропанол, 1-бутанол и вода с известными диэлектрическими и оптическими свойствами. При расчетах молекулярных структур, дипольных моментов основного и возбужденного состояний и поляризуемости использовался квантово-химический метод TDDFT с функционалом CAM-B3LYP в базисе 6-311G (d, p) в рамках программы Gamess (US). Полученное теоретическое значение эффективной диэлектрической проницаемости полости (около 13) хорошо согласуется с приведенным в литературе значением.
\end{abstract}

DOI: $10.21883 /$ OS.2019.04.47505.125-18

\section{Введение}

Исследование диэлектрических свойств полости макроциклических молекул, образующих комплексы включения с молекулами красителей, является актуальной задачей как с теоретической, так и с практической точек зрения. Изменение спектров поглощения и люминесценции хромофора, помещенного в полость кавитанда при комплексообразовании, может быть рассмотрено как сольватохромный сдвиг, связанный с изменением диэлектрических свойств окружения данного хромофора при его перемещении из раствора в оболочку из атомов данной макромолекулы. Теоретическое описание эффективной диэлектрической проницаемости макромолекулы - кавитанда позволяет прогнозировать величину сольватохромного сдвига спектра красителя при комплексообразовании, что представляет интерес при разработке оптических хемосенсоров, в которых в качестве рецепторного центра используется кавитанд.
Эффективное использование красителя в качестве нанозонда, позволяющего измерить локальное значение эффективной диэлектрической проницаемости кавитанда при образовании супрамолекулярного комплекса с данным красителем, продемонстрировано в работе [1]. При этом проводился экспериментальный подбор растворителя, сольватохромный сдвиг спектра растворенного красителя (родамина) в котором равен сдвигу, возникающему при комплексообразовании данного красителя с кукурбитурилом. Очевидно, что применение данного метода ограничено диапазоном растворимости красителя в растворителях, различающихся коэффициентом диэлектрической проницаемости.

С другой стороны, за последние несколько десятилетий хорошо разработаны методы расчета сольватохромных сдвигов оптического спектра на основе разновидностей модели Онзагера [2,3]. Применение этих методов к интерпретации сдвигов спектров красителя, связанных с перемещением его в новое локальное окружение, например при формировании супрамолекулярного комплекса, 
дает возможность определять локальный коэффициент диэлектрической проницаемости из теории.

При этом мы получаем возможность работать в широком диапазоне диэлектрической проницаемости, который выходит за пределы ограниченных возможностей экспериментальных измерений (например, вследствие отсутствия эталонного растворителя для данного красителя в требуемом диапазоне значений $\varepsilon$ ). Тогда эффективное значение $\varepsilon$ может быть получено непосредственно из измеренного значения сдвига оптического спектра красителя, помещенного в некоторое локальное окружение с измененными свойствами.

В настоящей работе мы апробировали этот подход для оценки диэлектрических свойств молекулы-,хозяина“ (кукурбит[7]урила) в супрамолекулярном комплексе включения по измеренному в эксперименте сдвигу оптического спектра поглощения молекулы-,гостя“ (красителя 1-(3-аммонийпропил)-4-[(Е)-2-(3,4-диметоксифинил)этенил]пиридиния) [4] при формировании комплекса. Экспериментальное и теоретическое исследование зависимости оптических спектров от положения хромофора красителей (диперхлораты 1- (2-аммониийэтил)4- [(Е) - 2 - (3, 4-диметоксифенил)этенил]пиридиния (D1), 1-(3-аммонийпропил)-4-[(E)-2-(3,4-диметоксифенил)этенил]пиридиния (D2) и 1-(5-аммонийпентил)-4-[(E)-2(3,4-диметоксифенил)этенил]пиридиния (D3), содержащих 2, 3 и 5 метиленовых групп соответственно) относительно полости кукурбит[7]урила (CВ[7]) было проведено в работе [5].

При этом, как показали наши расчеты, заряженный фрагмент хромофора красителя (катион пиридиния) занимает различное положение в комплексе относительно полости СВ[7] в зависимости от длины указанного $N$ заместителя, что отражается на оптических спектрах. В случае комплекса с D3 в полости кавитанда располагается аммониоалкильный $N$ заместитель, а заряженный пиридиновый фрагмент хромофора координируется по порталу и, таким образом, остается вне полости СВ[7] в окружении растворителя, поэтому существенного сольватохромного сдвига при комплексообразовании D3 с CВ[7] не происходит. В двух других случаях заряженный фрагмент хромофора красителя находится в полости, и сдвиг сольватохромной природы имеет место.

Природа гипсохромного сдвига для D1 и D2 при комплексообразовании связана с тем, что 1) дипольный момент молекулы в возбужденном состоянии (S1) больше, чем в основном ( $\mathrm{S} 0)$, и 2) полярность полости $\mathrm{CB}[7]$, как предполагается, меньше, чем полярность воды [5].

В настоящей работе сольватохромный сдвиг рассчитывался теоретически на основе модели Онзагера. Для параметризации модели был проведен эксперимент по исследованию сольватохромного эффекта для D2 в ряде растворителей, различающихся диэлектрической проницаемостью и показателем преломления. Другие характеристики красителя (оптимизированная структура молекулы, электрический дипольный момент в основном

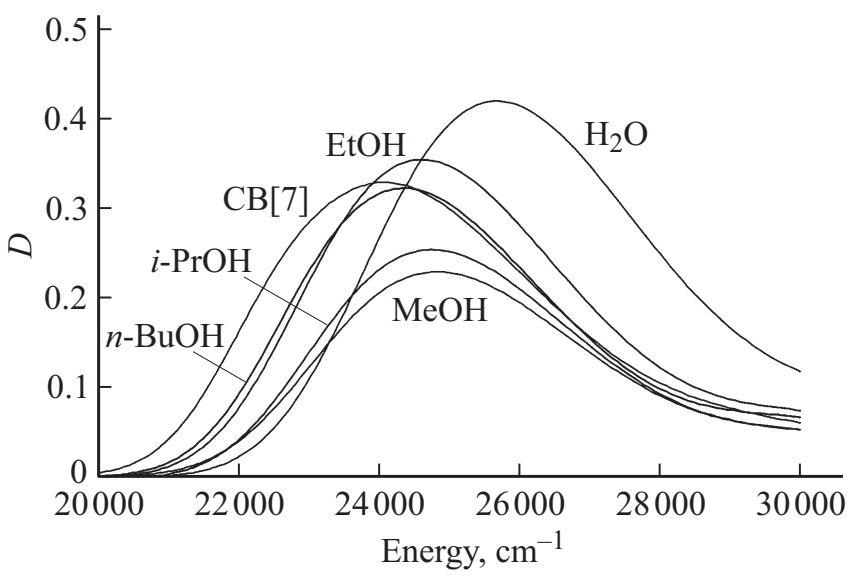

Рис. 1. Спектры поглощения красителя D2 в растворителях с различной диэлектрической проницаемостью.

и возбужденном состояниях, поляризуемость) определялись методами квантовой химии.

\section{Экспериментальная часть}

Был исследован стириловый краситель (СК) пиридинового ряда с $\mathrm{N}$-аммониоалкильным заместителем [6,7]: 1-(3-аммонийпропил)-4-[(Е)-2-(3,4-диметоксифинил)этенил]пиридиния (D2) (схема).

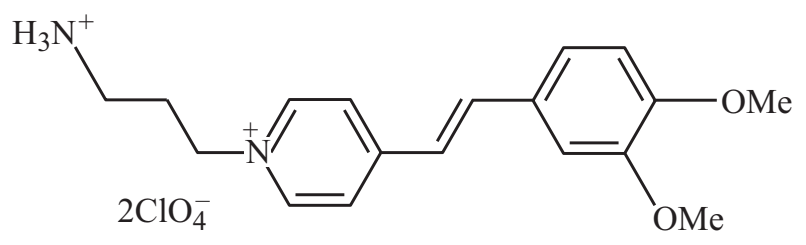

Схема. Структурная формула исследуемого стирилового красителя.

Были получены спектры поглощения растворов красителя D2 в растворителях, имеющих разную диэлектрическую проницаемость, а также комплекса включения D2, образуемого с $\mathrm{CB}[7])$. Спектры поглощения измеряли при $25^{\circ}$ на спектрофотометре Cary 4000 (Agilent Technology, США) в диапазоне 200-600 nm с шагом $1 \mathrm{~nm}$.

Для приготовления растворов использовались метанол, этанол, 1-пропанол, 1-бутанол и вода. Концентрация красителя D2 поддерживалась около $2 \cdot 10^{-5} \mathrm{~mol} \cdot \mathrm{L}^{-1}$. Спектры поглощения представлены на рис. 1.

Было показано, что с уменьшением полярности растворителя наблюдается все больший батохромный сдвиг. Величины сдвигов представлены в табл. 1. В таблицу также помещен экспериментально найденный сдвиг спектра при образовании комплекса включения D2CB[7] в водном растворе (рис. 2). В соответствии с нашим предположением данный сдвиг также имеет сольватохромную природу и связан с переносом хромофора 
Таблица 1. Экспериментальные значения сдвигов спектров поглощения $\mathrm{D} 2$ в различных растворителях относительно $\mathrm{H}_{2} \mathrm{O}$

\begin{tabular}{l|c|c|c|c}
\hline & $\varepsilon$ & $n$ & $\begin{array}{c}\text { Максимум } \\
\text { спектра } \\
\text { Роглощения, } \\
\text { ст }^{-1}\end{array}$ & $\begin{array}{c}\text { Сдвиг } \\
\text { спектра } \\
\text { относительно } \\
\mathrm{H}_{2} \mathrm{O}, \mathrm{cm}^{-1}\end{array}$ \\
\hline $\mathrm{H}_{2} \mathrm{O}$ & 80.1 & 1.333 & 25641 & 0 \\
$\mathrm{MeOH}$ & 32.6 & 1.327 & 24700 & 825 \\
$\mathrm{EtOH}$ & 24.3 & 1.361 & 24875 & 1070 \\
$i$-PrOH & 20.8 & 1.378 & 24630 & 887 \\
$n$ - $\mathrm{BuOH}$ & 17.8 & 1.399 & 24813 & 1251 \\
$\mathrm{CB}[7]$ & & & 24449 & 1700
\end{tabular}

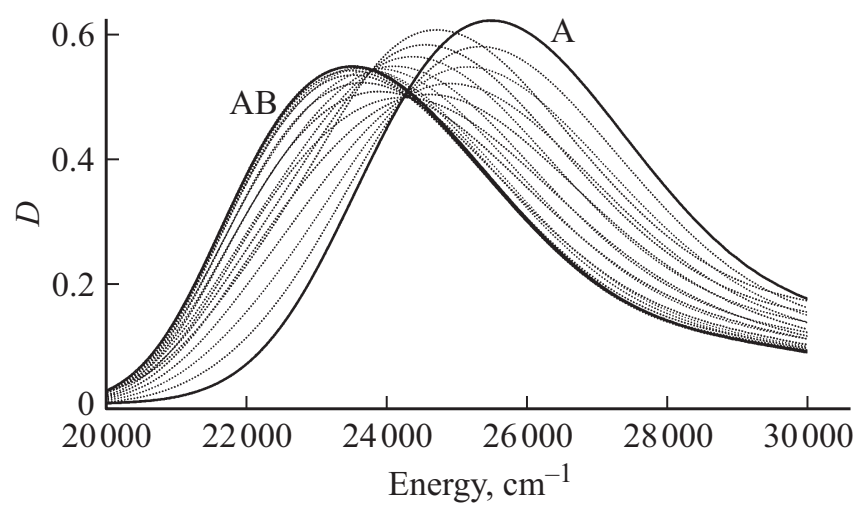

Рис. 2. Изменение спектра поглощения при образовании комплекса D2@CB[7], где A - краситель. АВ - комплекс.

D2 из водного окружения в полость $\mathrm{CB}[7]$, так что атомы полости выполняют ту же роль, что и атомы растворителя.

На основе полученных данных можно сделать качественный вывод, что эффективная полярность полости CВ[7] много меньше, чем у воды.

\section{Теоретическая часть}

Изменения в спектрах D2 в различных условиях могут быть поняты на основе хорошо разработанной теории Онзагера диполь-дипольного (универсального) взаимодействия СК с молекулами растворителя и с полостью $\mathrm{CB}[7][8-12]$.

В общем случае величина смещения частоты поглощения молекулы в растворе относительно молекулы в вакууме представляется в виде суммы вкладов дисперсионных, динамически поляризационных и ориентационноиндукционных взаимодействий [8-10]. Характерное время релаксации растворителя $\left(10^{-12} \mathrm{~s}\right)$ много меньше, чем время жизни возбужденного состояния $\left(10^{-9} \mathrm{~s}\right)[9$ 11], поэтому необходимо учитывать не только индукционную, но и ориентационную поляризацию. Напротив, вклад дисперсионных и динамических поляризационных взаимодействий предполагается пренебрежимо малым, так как поляризуемость молекулы в основном и возбужденном состояниях мало различается и может быть принята приблизительно постоянной в расчетах. Данное приближение подтверждается большим количеством экспериментальных данных $[11,12]$.

Суть эффекта в том, что ориентационно-поляризационные части дипольных моментов основного и возбужденного состояний меняются под действием реакционного поля со стороны растворителя. При этом изменяется и разность энергий основного и возбужденного состояний, что лежит в основе расчета сдвига спектральной линии. Модель Онзагера дает в данном случае довольно грубое приближение в связи с отличием формы рассматриваемых молекул от сферической, для которой разработана теория. Тем не менее большой и успешный опыт использования этой модели полуфеноменологического описания сольватохромных эффектов в существенно несферических молекулах, опубликованный во множестве работ [11,12], показывает прикладную ценность этой модели и в таких случаях - в качестве оценочного подхода, верно передающего физику эффекта. Накопленный опыт применения такого подхода явился аргументом использовать его в нашей работе.

Согласно теории, молекула хромофора может быть представлена в виде сферы радиуса $a$, в центре которой расположен дипольный момент. Важной особенностью рассматриваемого объекта - дикатиона - является наличие двух зарядов в растворе. То, что противоионом является $\mathrm{ClO}_{4}^{-}$- ион большого радиуса, позволяет обоснованно использовать предположение о том, что заряды дикатиона в растворе слабо скомпенсированы противоионами.

В указанном приближении энергетический сдвиг электронных состояний, вызванный неспецифическими взаимодействиями, как следует из теории реакционного поля Онзагера, имеет вид [10-12]

$$
\begin{gathered}
\boldsymbol{\mu}=\boldsymbol{\mu}_{0}+\alpha \mathbf{E}_{\mathrm{R}}, \\
\mathbf{E}_{\mathrm{R}}=\left(f_{\text {ind }}+f_{\text {or }}\right) \boldsymbol{\mu}, \\
f_{\text {or }}=\frac{2}{a^{3}}\left(\frac{\varepsilon-1}{2 \varepsilon+1}-\frac{n^{2}-1}{2 n^{2}+1}\right), \quad f_{\text {ind }}=\frac{2}{a^{3}}\left(\frac{n^{2}-1}{2 n^{2}+1}\right),
\end{gathered}
$$

где $\mathbf{E}_{\mathrm{R}}$ - реакционное поле Онзагера; $\boldsymbol{\mu}$ - дипольный момент растворенной молекулы; $f_{\text {int }}, f_{\text {or }}$ - индукционная и ориентационная функции отклика; $\boldsymbol{\mu}_{0}$ и $\alpha-$ дипольный момент и поляризуемость молекулы в вакууме соответственно, $a$ - радиус полости растворителя; $\varepsilon$ и $n$ - диэлектрическая проницаемость и показатель преломления растворителя. В данном случае, когда речь идет о дикатионе, под $\boldsymbol{\mu}$ и $\boldsymbol{\mu}_{0}$ следует понимать исключительно ориентационно-индукционную часть дипольного момента в растворе и в вакууме соответственно, которая инвариантна относительно преобразования координат, без зарядовой части, которая, согласно допущениям Онзагера, напрямую не влияет на эффективное значение 
реакционного поля. Тогда, согласно теории ОнзагераЛиптэя, изменение волнового числа определяется по формуле ([13], стр. 281)

$$
\begin{aligned}
h c \Delta v & =-\frac{1}{2}\left[\frac{2}{a^{3}}\left(\frac{n^{2}-1}{2 n^{2}+1}\right)\right]\left(\mu_{e}-\mu_{g}\right)^{2} \\
\times & {\left[1-\frac{2}{a^{3}}\left(\frac{n^{2}-1}{2 n^{2}+1}\right) \alpha\right]^{-1}-\frac{2\left(\mu_{e}-\mu_{g}\right)}{a^{3}}\left(\frac{\varepsilon-1}{2 \varepsilon+1}\right) } \\
\times & {\left[1-\frac{2}{a^{3}}\left(\frac{\varepsilon-1}{2 \varepsilon+1}\right) \alpha\right]^{-1} \mu_{g} . }
\end{aligned}
$$

Здесь $\mu_{e}, \mu_{g}$ - дипольные моменты возбужденного и основного состояний, которые здесь предполагаются параллельными, что отвечает рассматриваемому случаю.

В расчетах сольватохромных спектральных сдвигов по формуле (1) величины $\varepsilon$ и $n$ были взяты из справочника [14] для соответствующих растворителей; дипольные моменты основного и возбужденного состояний, а также поляризуемость красителя определялись методами квантовой химии.

Для квантово-химических вычислений энергий возбужденного состояния и равновесной геометрии (рис. 3) использовался квантово-химический метод TDDFT с функционалом CAM-B3LYP в базисе 6-311G (d,p) в рамках программы Gamess (US) [15-17]. Ориентационно-индукционные части дипольных моментов были вычислены для основного (39.7 D) и возбужденного (34.3 D) состояний дикатиона.

Хотя сами заряды в приближении Онзагера непосредственно не влияют на сдвиг энергии перехода ([10], стр. 24), косвенное влияние - через поляризуемость молекулы - довольно велико и было учтено в квантовохимическом расчете индукционно-ориентационной части дипольного момента. При этом индуцируется момент, направленный приблизительно вдоль главной оси молекулы (имеющей вытянутую форму), поскольку и сами заряды дикатиона расположены примерно вдоль нее. Также для дальнейших рассуждений важно отметить, что ориентационно-индукционная часть дипольного момента ориентирована вдоль главной оси молекулы (рис. 4).

В модели Онзагера полязизуемость молекулы, которая учитывает действие реакционного поля на молекулу, считающуюся изотропной, рассматривается как скаляр. Для объекта с анизотропной поляризуемостью, как в данном случае, индуцированный средой дипольный момент вдоль оси х можно записать в виде

$$
\mu_{i, x}=\alpha_{x x} E_{x}+\alpha_{x y} E_{y}+\alpha_{x z} E_{z},
$$

где $\alpha_{j k}$ - тензор поляризуемости, $\mathbf{E}-$ электрическое поле. Аналогично для $y$ - и $z$-компонентов момента. Наиболее просто это выражение записывается для диагонализированной матрицы поляризуемости $\alpha_{D}$ :

$$
\Delta \mu_{i, x}=\alpha_{D, x x} E_{x} .
$$

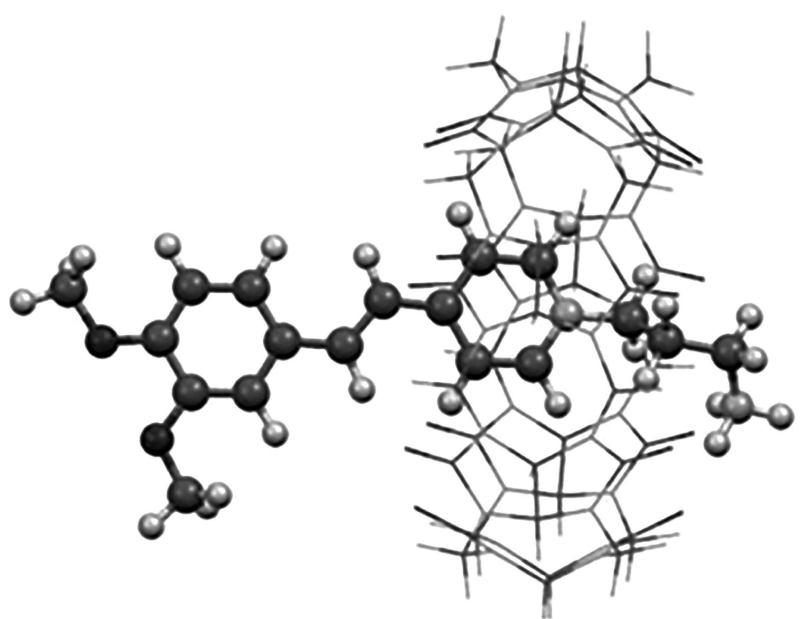

Рис. 3. Оптимизированная структура комплекса включения D2@CB[7]. Кавитанд располагается над пиридиниевым фрагментом молекулы.

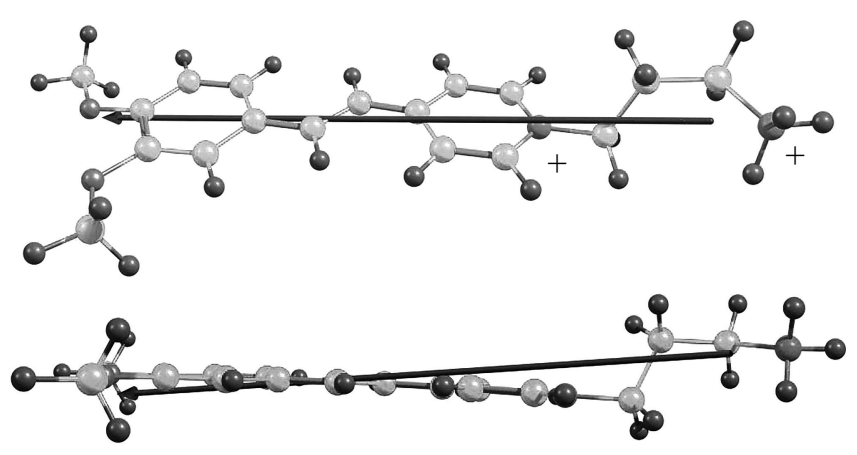

Рис. 4. Стрелкой указана ориентационно-индукционная часть дипольного момента дикатиона $\mu_{0}$ в двух проекциях.

Модуль полного приращения момента может быть найден по формуле

$$
\Delta \mu_{i}=\sqrt{\alpha_{D, x x}^{2} E_{x}^{2}+\alpha_{D, y y}^{2} E_{y}^{2}+\alpha_{D, z z}^{2} E_{z}^{2}} .
$$

C учетом того, что $\mathbf{E}=\mathbf{E}_{\mathrm{R}}-$ реакционное поле, индуцированное в среде ,затравочным“ моментом $\boldsymbol{\mu}_{0}$, направленным приблизительно коллинеарно главной оси дикатиона (рис. 4), и с учетом малости $y y$ - и $z z$-компонент тензора поляризуемости получаем

$$
\Delta \mu_{i} \approx \alpha_{D, x x} \mathbf{E}_{\mathrm{R}}
$$

откуда для эффективной поляризуемости имеем оценку

$$
\alpha \approx \alpha_{D, x x}
$$

Дипольные моменты основного и возбужденного состояний дикатиона, а также его поляризуемость были вычислены квантово-химическиим методом TDDFT с функционалом CAM-B3LYP в базисе 6-311G (d, p) в рамках программы Gamess (US) [15-17]. Рассчитанные таким образом индукционно-ориентационные части 


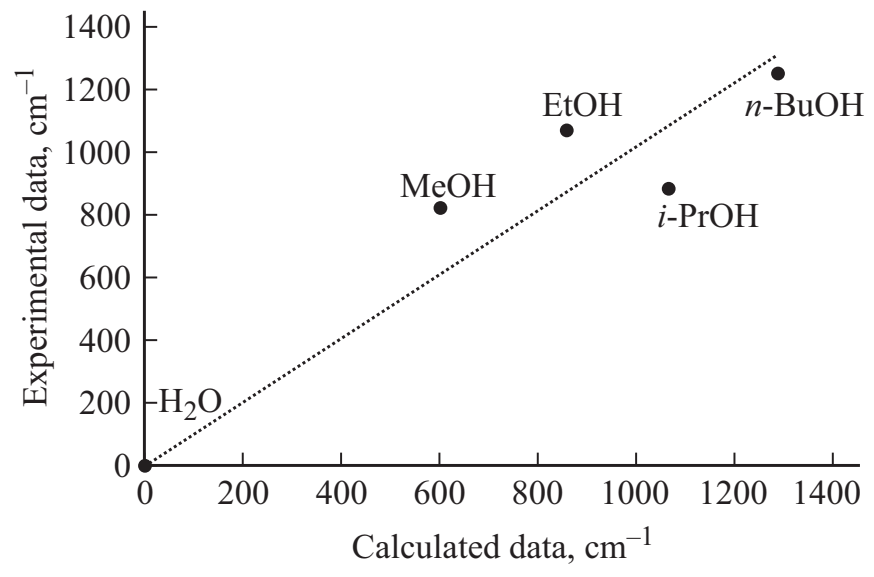

Рис. 5. Результат фитинга рассчитанных по формуле (1) и экспериментальных сдвигов спектра красителя D2 (табл. 1) в разных растворителях, отвечающих характерному размеру молекулы $a \approx 5.6$.

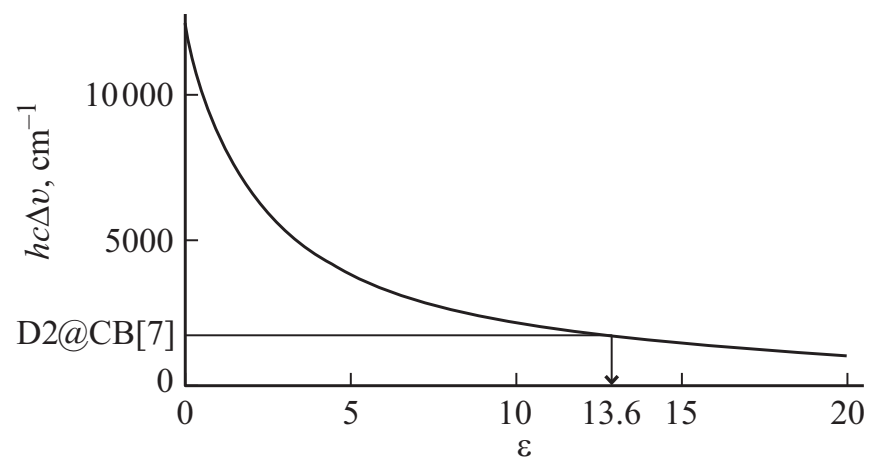

Рис. 6. Определение эффективной диэлектрической проницаемости полости СВ[7] по теоретической зависимости сольватохромного сдвига (1) от $\varepsilon$.

дипольных моментов основного и возбужденного состояний дикатиона равны соответственно $\mu_{g} \approx 39.7 \mathrm{D}$, $\mu_{e} \approx 34,3 \mathrm{D}$.

Диагонализированная матрица поляризуемости (в атомный единицах, а.u.) имеет вид

$$
\alpha_{D}=\left(\begin{array}{ccc}
609.3 & 0 & 0 \\
0 & 195.4 & 0 \\
0 & 0 & 114.6
\end{array}\right)
$$

откуда, согласно (3), $\alpha \approx 609$ а.u.

Формула (1) позволяет связать экспериментально полученные сдвиги спектров в разных растворителях относительно вакуума с учетом известных значений $\varepsilon$ и $n$ (табл. 1). При этом подгоночным параметром является эффективный радиус молекулы $a$. Проведенный фитинг показал, что наилучшее согласование экспериментальных данных $(\Delta v, \varepsilon$ и $n)$ достигается при выборе размера молекулы $a \approx 5.6 \AA$ (рис. 5 ).

С другой стороны, эффективный размер молекулы D2 был оценен на основе вычисления объема молекулы
Таблица 2. Расчетные и экспериментальные значения сдвигов спектров поглощения D2 в различных растворителях относительно $\mathrm{H}_{2} \mathrm{O}$

\begin{tabular}{|c|c|c|c|c|}
\hline Растворитель & $\varepsilon$ & $n$ & $\begin{array}{c}\text { Расчетные } \\
\text { сдвиги } \\
\text { спектра } \\
\text { относительно } \\
\mathrm{H}_{2} \mathrm{O}, \mathrm{cm}^{-1}\end{array}$ & $\begin{array}{c}\text { Эксперимен- } \\
\text { тальные } \\
\text { сдвиги } \\
\text { спектра } \\
\text { относительно } \\
\mathrm{H}_{2} \mathrm{O}, \mathrm{cm}^{-1}\end{array}$ \\
\hline $\mathrm{H}_{2} \mathrm{O}$ & 80.1 & 1.333 & 0 & 0 \\
\hline $\mathrm{MeOH}$ & 32.6 & 1.327 & 605 & 825 \\
\hline $\mathrm{EtOH}$ & 24.3 & 1.361 & 859 & 1070 \\
\hline$i-\mathrm{PrOH}$ & 20.8 & 1.378 & 1068 & 887 \\
\hline$n-\mathrm{BuOH}$ & 17.8 & 1.399 & 1291 & 1251 \\
\hline
\end{tabular}

красителя (с учетом ван-дер-ваальсовых радиусов атомов и их взаимных перекрываний). Если принять вандер-ваальсовы радиусы равными: для атомов $\mathrm{H}, \mathrm{C}, \mathrm{O}$ и $\mathrm{N}-1.2,1.7,1.52$ и $1.55 \AA$ [18], то объем молекулы составляет $296 \AA^{3}$. Данный объем отвечает сфере с радиусом 4.2 А. Если характерный размер молекулы оценивать по ее полудлине, получим около $8 \AA$ А. Таким образом, найденное из фитинга значение указанного параметра хорошо согласуется с размерами молекулы, полученными из квантово-химических расчетов.

Подстановка $a \approx 5.6 \AA$ позволяет определять сдвиги спектра для произвольных значений $\varepsilon$ и $n$ по формуле (1). В результате расчетов были получены сдвиги спектров поглощения D2 в растворителях с различной диэлектрической проницаемостью относительно $\mathrm{H}_{2} \mathrm{O}$. Результаты в сравнении с экспериментом представлены в табл. 2.

Можно сделать вывод, что расчетный метод способен предсказывать смещение спектра поглощения данного красителя, окруженного средами разной полярности. Наоборот, зная из эксперимента смещение спектра, можно определить полярность окружения. Это позволяет использовать краситель в качестве зонда для исследования локальных характеристик матрицы, в которую он помещен.

В частности, зная экспериментальное смещение спектра красителя при помещении его хромофора в полость CВ[7] (табл. 1), можно оценить эффективную диэлектрическую проницаемость полости. Расчет производился при характерном значении показателя преломления $n=1.3$ и привел к диэлектрической проницаемости для полости $\varepsilon \approx 13.6$ (рис. 6 ).

В литературе имеются данные об экспериментальных исследованиях полости кукурбитурила [19], согласно которым диэлектрическая проницаемость полости СВ[7] сравнима с октанолом $(\varepsilon=10.3$ при $n=1.17)$, что сопоставимо с полученным в результате применения расчетного метода значением. (Заметим также, что при подстановке $n=1.17$ в формулу (1) полученное из 
эксперимента значение сольватохромного сдвига достигается при $\varepsilon \approx 13.6$.)

\section{Заключение}

В работе предложен новый метод оценки диэлектрических свойств молекулы-,хозяина“ в супрамолекулярном комплексе включения по измеренному в эксперименте сдвигу оптического спектра поглощения молекулы„гостя“ (красителя) при формировании указанного комплекса. Спектральный сдвиг трактуется как сольватохромный, а для получения значения эффективной диэлектрической проницаемости применяется теория Онзагера, параметры для которой получаются из квантовохимических расчетов (дипольные моменты красителя в основном и возбужденном состояниях и его поляризуемость).

Модель была откалибрована на экспериментальных данных по сольватохромным сдвигам данного красителя в разных растворителях. При этом верифицировался такой параметр модели Онзагера как эффективный радиус молекулы-,„гостя“. Тот же радиус оценивался методами квантовой химии с учетом ван-дер-ваальсовых радиусов атомов. В работе показано, что квантово-химическая оценка радиуса молекулы (5.6 $\AA$ ) хорошо согласуется с величиной радиуса, полученного из калибровки формулы Онзагера-Липтэя по экспериментальным сольватохромным сдвигам. Найденное значение диэлектрической проницаемости полости $\mathrm{CB}[7](\varepsilon \approx 13.6)$ согласуется с указанной величиной $(\varepsilon \approx 10.3)$, полученной с использованием другого красителя (родамина) другим, чисто экспериментальным методом [19]: проводился экспериментальный подбор растворителя, сольватохромный сдвиг спектра красителя в котором наиболее близок к сдвигу, возникающему при комплексообразовании данного красителя с CB[7]. Применение теоретического метода позволяет использовать более узкую выборку растворителей только для калибровки рабочей формулы и исключает ситуацию, когда подбор растворителя, дающего сдвиг, наиболее близкий к искомому, невозможен (малая растворимость красителя в любых средах с требуемой полярностью).

Предложенный метод может использоваться для зондирования локальных диэлектрических свойств наноструктурированных систем в различных условиях. Например, в работе [20] было показано наличие спектрального сдвига при функционализации наноалмаза фотохромными соединениями из класса спиропиранов и спирооксазинов. Такой спектральный сдвиг, по крайней мере частично, может быть интерпретирован как сольватохромный с учетом того, что локальные свойства растворителя для сорбированного красителя изменяются в присутствии поверхности частицы; при этом наличие калибровочной кривой сольватохромного сдвига дает возможность оценить диэлектрические свойства на поверхности сорбента.
Модель Онзагера - первое приближение к решению задачи сольватохромного сдвига для удлиненной молекулы. Однако опыт применения этого приближения к интерпретации сольватохромного сдвига, наблюдаемого для органических молекул сложной формы в растворителях с различной диэлектрической проницаемостью $[11,12]$, говорит о феноменологическом значении такого подхода, позволяющего, по крайней мере полуколичественно, интерпретировать экспериментальные данные. Простота этого подхода является его преимуществом по сравнению с гораздо более сложными методами учета растворителя квантово-химическими методами, которые при этом также содержат сильные допущения по поводу распределения молекул растворителя относительно хромофора. Развитие континуальной модели, обобщающей подход Онзагера на молекулы сложной формы, продолжает оставаться актуальной задачей.

Авторы благодарны д.ф.-м.н. профессору В.Г. Плотникову за обсуждение теоретических моделей сольватохромного спектрального сдвига, к.х.н. В.Г. Авакяну и к.Х.н. К.Г. Комаровой за консультации в области квантово-химических расчетов, а также член-корр. д.х.н. С.П. Громову за обеспечение возможности проведения экспериментальной части работы. Работа выполнена при поддержке Министерства науки и высшего образования в рамках выполнения работ по Государственному заданию ФНИЦ „Кристаллография и фотоника“" РАН в части квантово-химических расчетов и эксперимента, а также при поддержке программы ПКС НИЯУ МИФИ в части обоснования параметра фитинга по модели Онзагера-Липтэя.

\section{Список литературы}

[1] Magde D., Wong R., Seybold P.G. // Photochem. Photobiol. 2002. V. 75. P. 327.

[2] Onsager L. // J. Am. Chem. Soc. 1936. V. 58. N 8. P. 1486.

[3] Плотников В.Г., Сажников В.А., Алфимов М.В. // Химия высоких энергий. 2007. Т. 41. № 5. С. 1.

[4] Громов С.П., Ведерников А.И., Сазонов С.К., Лобова Н.А., Алфимов М.В. Патент РФ № 2383571. БИ 2010. № 7.

[5] Авакян В.Г, Степко А.С., Лобова Н.А., Грушникова Е.Ю., Сазонов С.К., Громов С.П., Плотников В.Г., ЛебедевСтепанов П.В. // ДАН. 2017. Т. 476. № 4. С. 406.

[6] Ведерников А.И., Кузьмина Л.Г., Сазонов С.К., Лобова Н.А., Логинов П.С., Чураков А.В., Стреленко Ю.А., Ховард Джс.А.К., Алфимов М.В., Громов С.П. // Изв. АН. Сер. хим. 2007. № 9. С. 1797.

[7] Gromov S.P., Vedernikov A.I., Kuz'mina L.G., Kondratuk D.V., Sazonov S.K., Strelenko Y.A., Alfimov M.V., Howard J.A.K. // Eur. J. Org. Chem. 2010. N 13. P. 2587.

[8] Бахшиев Н.Г. Фотофизика диполь-дипольных взаимодействий: Процессы сольватации и комплексообразования. СПб.: Изд-во СПб. гос. университета, 2006. 500 с.

[9] Reichardt C. // Chem. Rev. 1994. V. 94. N 8. P. 2319.

[10] Бахшиев Н.Г. Спектроскопия межмолекулярных взаимодействий. Л.: Наука, 1972. 264 с. 
[11] Komarov P.V., Plotnikov V.G. // Intern. J. Quant. Chem. 2012. V. 112. P. 3039.

[12] Komarova K.G., Sakipov S.N., Plotnikov V.G., Alfimov M.V. // J. Luminesc. 2015. V. 164. P. 57.

[13] Modern Quantum Chemistry. Part II: Interactions. / Ed. by Liptay W., Sinanoglu O. New York: Academic Press, 1965. P. 282. (Chapter 5).

[14] Rumble J. CRC Handbook of Chemistry and Physics. 98th Edition. CRC Press, 2017.

[15] Schmidt M.W., Baldridge K.K., Boatz J.A., Elbert S.T., Gordon M.S., Jensen J.H., Koseki S., Matsunaga N., Nguyen K.A., Su S., Windus T.L., Dupuis M., Montgomery J.A. // J. Comput. Chem. 1993. V. 14. P. 1347.

[16] Gordon M.S., Schmidt M.W. Theory and Applications of Computational Chemistry: The First Forty Years. / Ed. by Dykstra C.E., Frenking G., Kim K.S., Scuseria G.E. Amsterdam: Elsevier, 2005. P. 1167 (Ch. 41).

[17] Gordon M.S. Gamess (US). [Электронный ресурс]. Режим доступа: https://www.msg.chem.iastate.edu/index.html

[18] Bondi A. // The J. Phus. Chem. 1964. V. 68. N 3. P. 441.

[19] Assaf K.I., Nau W.M. // Chem. Soc. Rev. 2015. V. 44. P. 394.

[20] Венидиктова О.В., Валова Т.М., Барачевский В.А., Айт А.О., Лебедев-Степанов П.В., Вуль А.Я., Кольцова Л.С., Шиенок А.И., Зайченко Н.Л. // Опт. и спектр. 2017. T. 122. № 5. С. 751. 